339); Hub $1(77 \% ; n=72 / 93)$, Hub 2 (73\%; $n=97 / 133)$, Hub 3 (79\%; $\mathrm{n}=89 / 113)$. For non-attending patients, a medication review was conducted in their absence by the prescriber for most $(94 \% ; n=$ 74/81) patients (see table 1 and Figure 1).

In January 2020, reassessment of attendance rates for Hub 1 (subsample), in January $2020(\mathrm{n}=91)$ which showed attendance had increased to $86 \%(n=78 / 91)$. All $(100 \% n=13)$ patients who did not attend for the prescriber review in person, had a medication review in their absence. In addition, the reasons for nonattendance were discussed with the patient and their keyworker, following which they were booked for a subsequent appointment.

Conclusion. Nonattendance at clinical appointments causes a significant financial burden across the NHS. It was fantastic to see that the QIP improved patient attendance rates and this was sustained and improved, over a year later. Serial non-attenders may need an enhanced strategy.

\section{Exploring the views of young people with autism spectrum disorder (ASD) on how to improve medical consultations}

\section{Alexander Mayor}

Leicestershire Partnership Trust, University of Exeter Medical School, Barnet, Enfield and Haringey Mental Health Trust, Camden and Islington NHS Foundation Trust

doi: 10.1192/bjo.2021.553

Aims. The aim of this study was to explore experiences of consultations with non-specialist health professionals in a group of young people with ASD and their parents and what they considered would help to improve these interactions.

Background. Research has shown that general awareness around autism is poor among the general medical community and this can result in poor communication during medical consultations. Method. An opportunistic group of 4 young people with ASD and 8 parents from a local support group in Exeter were interviewed in an informal environment about their experiences of healthcare consultations after seeking verbal consent.

Result. Among the 8 parents interviewed the themes emerging were a deep lack of understanding and awareness among medical staff of the challenges faced by individuals with autism, the importance of the doctor-patient relationship to allow children to open up to healthcare professionals, and the need for all children to be respected as individuals.

Among the 4 young people (13 to 19 years) with ASD interviewed they identified significant anxiety around waiting for appointments and expressed a desire for a distractor to relieve stress, questions posed by clinicians were often vague and should be clearer, $3 \mathrm{D}$ models/mannequins could be useful to support understanding of anatomy and physiology, patience and a calm demeanour were vital with a quiet clinical environment to avoid distraction, time for mental preparation is important and efforts should be made to avoid delays or cancellation of appointments where possible.

An interactive website was generated in the light of feedback from the client group and their parents, aiming to educate clinicians regarding the challenges faced by this client group and provide a guide suggesting how to facilitate effective consultation through the use of simple techniques to promote engagement/ reduce anxiety in the clinical environment.

Conclusion. Simple changes to the approach to consultation with clients with ASD - a quiet consultation space, no delays and better communication - could reduce stress and promote positive interactions with a beneficial effect on healthcare delivery for this client group.
No time to die: improving response to emergency scenarios in the 136 suite

Rebecca McKnight* and Nicola Combs

Greater Manchester Mental Health Trust

${ }^{\star}$ Corresponding author.

doi: 10.1192/bjo.2021.554

Aims. Improve confidence and experience of trainees performing preliminary medical reviews in the 136 suite.

Improve patient safety by increasing trainee's confidence in responding to emergency scenarios, including crash calls of patients in the 136 Suite.

To orientate trainees to the 136 suite and the emergency crash equipment, in order to better prepare trainees for emergency scenarios.

Background. The authors encountered a crash call in the 136 suite, in which a patient had concealed an opiate overdose. The patient was successfully resuscitated but concerns were raised by the junior doctors that they were unaware of what or where the emergency equipment was kept in the 136 suite. Following a debrief session, we established that junior doctors needed more orientation to the 136 suite and more teaching on performing preliminary medical reviews and responding to emergency situations. Method. Trainees, were asked to complete an anonymous, qualitative questionnaire with 16 questions asking about their confidence to respond to emergency situations in the 136 suite.

Based on the feedback, an interactive teaching session was delivered two weeks later. The session covered a structured approach on how to perform a preliminary medical review and scenario-based teaching on emergency situations. Trainees were then shown the 136 facility, introduced to the lead nurse and shown the emergency crash equipment and drugs stores.

Trainees were then re-consulted, with the same questionnaire to ascertain whether confidence and knowledge had increased.

Result. Following initial induction, only $25 \%$ of trainees felt confident performing 136 Suite preliminary reviews. 50\% of trainees had encountered crash calls at Park House Hospital, however 93\% did not receive orientation of emergency equipment locations. Only $44 \%$ of trainees felt confident managing a crash call; reasons included feeling 'rusty, little recent experience, not being familiar with the equipment'.

Post-interactive teaching session, $89 \%$ now felt confident performing 136 Suite preliminary reviews. 100\% knew where the crash equipment was located in the 136 Suite.

Conclusion. Trainees should receive a robust induction on how to perform 136 preliminary reviews and have orientation of the facility, including crash equipment during induction

Trainees require refresher training in addition to their basic life support training on common emergency scenarios encountered in psychiatric hospitals.

A resuscitation skills training session is being organised for new trainees and hopefully incorporated into each forthcoming rotation.

\section{Dublin's homeless crisis - is this reflected in emergency department psychiatry referrals?}

Aoibheann McLoughlin ${ }^{1 *}$, Anna Feeney ${ }^{2}$ and John Cooney ${ }^{1}$

${ }^{1}$ St. James's Hospital and ${ }^{2}$ St. Patrick's Hospital

${ }^{\star}$ Corresponding author.

doi: 10.1192/bjo.2021.555

Aims. This study seeks to explore the prevalence and impact of homelessness in an adult sample of psychiatry referrals over a 